\title{
Comparative genomics and quantitative proteomics reveal differentially produced proteins underlying virulence and host specificity in Bacillus thuringiensis
}

\author{
Yury Malovichko \\ Laboratory for Proteomics of Supra- \\ Organismal Systems \\ All-Russian Research Institute of \\ Agricultural Microbiology \\ Saint Petersburg, Russia \\ yu.malovichko@arriam.ru \\ Daria Gorbach \\ Department of Biochemistry \\ St. Petersburg State University \\ Saint Peterburg, Russia \\ daria.gorba4@yandex.ru \\ Maria Belousova \\ Laboratory for Proteomics of Supra- \\ Organismal Systems \\ All-Russian Research Institute of \\ Agricultural Microbiology \\ Saint Petersburg, Russia \\ m.belousova@arriam.ru \\ Ekaterina Romanovskaya \\ Department of Biochemistry \\ St. Petersburg State University \\ Saint Peterburg, Russia \\ e.romanovskaya@spbu.ru
}

\author{
Elena Lukasheva \\ Department of Biochemistry \\ St. Petersburg State University \\ Saint Peterburg, Russia \\ elena_lukasheva@mail.ru \\ Christian Ihling \\ Department of Pharmaceutical \\ Chemistry and Bioanalytics \\ Institute of Pharmacy, Martin-Luther \\ Universität Halle-Wittenberg \\ Halle, Germany \\ christian.ihling@pharmazie.uni- \\ halle.de \\ Andrej Frolov \\ Department of Biochemistry \\ St. Petersburg State University \\ Saint Petersburg, Russia \\ Department of Pharmaceutical \\ Chemistry and Bioanalytics \\ Institute of Pharmacy, Martin-Luther \\ Universität Halle-Wittenberg \\ Halle, Germany \\ Andrej.Frolov@ipb-halle.de
}

\author{
Anton Nizhnikov \\ Laboratory for Proteomics of Supra- \\ Organismal Systems \\ All-Russian Research Institute of \\ Agricultural Microbiology \\ Saint Petersburg, Russia \\ Department of Genetics and \\ Biotechnology \\ St. Petersburg State University \\ Saint Peterburg, Russia \\ a.nizhnikov@arriam.ru \\ Kirill Antonets \\ Laboratory for Proteomics of Supra- \\ Organismal Systems \\ All-Russian Research Institute of \\ Agricultural Microbiology \\ Saint Petersburg, Russia \\ Department of Genetics and \\ Biotechnology \\ St. Petersburg State University \\ Saint Peterburg, Russia \\ k.antonets@arriam.ru
}

\begin{abstract}
Bacillus thuringiensis is a Gram-positive sporeforming bacterium known for its insecticidal activities. Although its features of high virulence and exceptional host specificity are wiely known and have conditioned its use as a source of novel biopesticides, molecular mechanisms underlying these traits remain elusive and are usually attributed to its repertoire of proteinaceous toxins. In this work we used combined proteogenomic approach to dissect $<>$. We used three different strains of $B$. thuringiensis belonging to the var. thuringiensis, darmstadtiensis and israeliensis and one derivate of $B$. thuringiensis var israeliensis, which lost the ability to produce Cry-toxins. By using hybrid Oxford Nanopore and Illumina sequencing we achieved replicon-level genome assemblies of the studied strains which, upon annotation, facilitated comparison of virulence factor repertoires and putative reasons of virulence loss in derivate strain, Further application of quantitative HPLC-Orbitrap-MS and proteome level-comparison between vegetative and sporulating cultures allowed us to identify the proteins, which were differentially produced in the strains at each stage. While proteins differentially produced at vegetative stage related mostly to cell metabolism with few virulence factors captured, those identified in spores included different spore coat proteins, flotillin-like proteins involved in in cell differentiation, exosporium proteins and a wide set of virulence factors including proteinaceous Cry toxins. Taking together the data obtained in this study indicate the impact of the accessory genes produced at various stages of colony development on pathogenicity-associated phenotypic traits in strains with different host ranges. To our knowledge, this is the first proteogenomic study of $\boldsymbol{B}$. thuringiensis aimed to compare both genomic and proteomic profiles between serovars.
\end{abstract}

Keywords - bacillus thuringiensis, biopesticides, toxins, genomics, proteomics, pathogenicity, virulence, specificity, nanopore, illumina, orbitrap

\section{Introduction}

Bacillus thuringiensis, commonly referred to as Bt, is a spore-forming bacterium of Firmicutes phylum mostly known as a pathogen of various invertebrates, especially insects [1]. A peculiar feature of this bacterium is that, despite a vast range of potential hosts, specificity of particular strains is usually confined to a usually narrow set of invertebrate species. Traditinally such specificity is attributed to proteinaceous toxins produced either by vegetative cells (e.g. Vip and Sip toxins) or at the onset of sporulation, of which three-domain toxins are the most well-known [2]. This paradigm, however, does not take into account numerous auxiliary virulence factors, such as chitinases, metalloproteases and phospholipases, which emerge to gain growing attention of microbiologists [3]. The abundance of this factors and distribution of respective genes among existent $B t$ strains confounds the exploration of molecular mechanisms modulatin $B t$ host specificity. Although genomics and other omics techniques are being widely used in $B t$ studies, none of them have incorporated these approaches to compare virulence factor repertoires across strains or serovars. In this work we perform whole genome sequencing (WGS) of four $B t$ strains belonging to three different serovars followed by quantitative mass-spectrometry (MS) of respective vegetative and sporulating cultures. Our results indicate a set of proteins which may be related to specific virulence features known for the tested strains and patterns of their production, 


\section{Materials And Methods}

Three virulent $B t$ strains belonging to darmstadtiensis (strain 109/25), israeliensis (strain 800/3) and thuringiensis (strain 800/15) serovars and an avirulent derivate of var. israeliensis strain were used in this work. Genomic DNA was extracted from vegetative bacterial cultures and sequenced with Illumina HiSeq X (Illumina, San Diego, CA, USA) and MinION (Oxford Nanopore Technologies, Oxford, UK). Genome assembly was performed using Flye with ON-based error correction was performed by four-wise launching of Minimap2 and Racon followed by Medaka and llumina-based assembly correction performed with Pilon. Quality control was performed using QUAST, and assembly graphs were visualized using Bondage. Obtained assemblies were annotated using Prokka with Bacillus-related protein seqeunces from NCBI IPG as a database. Genome-level contig alignment was performed using MUMmer.

For proteomic assays, total protein fraction was extracted from both vegetative cells grown on LB medium and sporulating culture grown on T3 medium to induce spore formation, Qualitative proteome analysis was performed using electrospray quadruple mass-spectrometry coupled with fast performance liquid chromatography (FPLC-ESI-MS) of spots selected by two-dimensional difference gel electrophoresis (2D-DIGE). For quantitative analysis, a highperformance liquid chromatography/Orbitrap electrospray mass-spectrometry (HPLC-Orbitrap-MS) was used. Resulting spectra were annotated using Progenesis QI and Perseussoftware. Acquired data were preprocessed, normalized and imputed at protein level using Msnbase $\mathrm{R}$ package. Differential expression was assessed by independent use of two R packages, limma and MsqRob. Proteome-wide functional annotation was performed using eggNOG v2.0 with subsequent over-representaion assessment in differentially expressed gene subsets using topGO and BOG R packages.

\section{Results}

Genomes of all four strains used in this work were assembled up to replicon level. Subsequent annotation revealed all of these strains possessed genes encoding chitinases, phospholipases $\mathrm{C}$ and $\mathrm{CalY}$ metalloproteases, while distribution of several other virulence factors such as ColB, NlpC and NprB were confined to particular strains. Also, the Cry gene repertoire for each strain was evaluated. It appeared that strain $800 / 15$ bear genes for Cry1Ba1 and Cry1Ab12, strain 109/25 possess genes encoding Cry1Ea10 toxin and strain 800/3 genome encodes Cry4Ba1, Cry4Aa2 and Cry10Aa3 according to Bt Nomenclature []. Pathogenicity loss in the avirulent strain was attributed to the absence of the Cry-encoding plasmid present in the progenitor strain.

Qualitative proteomic assay revealed 21 differentially produced protein among four strains in question. Further quantitative HPLC-Orbitrap-MS showed that all four strains show similar patterns of differences between vegetative and sporulating cells which reflect processes underlying cll compaction and spore formation with a median of 20 proteins upregulated in vegetative cells and 30 proteins upregulated in spores. At the same time, interstrain comparisons at the respective stages pointed out differences potentially involved in pathogenicity phenotypes. Proteins differentially produced at the vegetative stage mostly involve cellular metabolism enzymes with only few virulence factors captured, including flagellins and virulence-related metalloproteases. Those present in the spores, however, are enriched with virulence determinants including Cry toxins as well as different proteins composing spore coat, exosporium and cellular membranes. Taken together, obtained data not only revealed changes in the B. thuringiensis proteomes associated with vegetative cellsspore transition, but also shed light on the differences between the strains at both stages, which might help to better understand mechanisms of host specificity.

\section{ACKNOWLEDGMENT}

This work was supported by the Russian Foundation for Basic Research, Project 20-316-70020.

\section{REFERENCE}

[1] B Raymond, PR Johnston, C Nielsen-LeRoux, D Lereclus, N Crickmore. Bacillus thuringiensis: an impotent pathogen?. Trends Microbiol. 2010;18(5):189-194. doi:10.1016/j.tim.2010.02.006

[2] L Palma, D Muñoz, C Berry, J Murillo, P Caballero. Bacillus thuringiensis toxins: an overview of their biocidal activity. Toxins (Basel). 2014;6(12):3296-3325. Published 2014 Dec 11. doi:10.3390/toxins6123296

[3] YV Malovichko, AA Nizhnikov, KS Antonets. Repertoire of the Bacillus thuringiensis Virulence Factors Unrelated to Major Classes of Protein Toxins and Its Role in Specificity of Host-Pathogen Interactions. Toxins (Basel). 2019;11(6):347. Published 2019 Jun 17. doi:10.3390/toxins11060347 